\title{
Una rilettura interculturale del concetto di spazio potenziale di Donald Winnicott. Analisi di un'esperienza educativa italiana
}

\section{Uma releitura intercultural do conceito de espaço potencial de Donald Winnicott: análise de uma experiência educacional italiana}

\section{The potential space of Donald Winnicott in intercultural education: an italian early education project}

\author{
Anna Aluffi Pentini*
}

\begin{abstract}
SOMMARIO
In questo contributo si vuole dare conto della situazione italiana per quanto riguarda l'approccio educativo alla prima infanzia, e descrivere un progetto interculturale ${ }^{1}$, ideato, realizzato, monitorato e sostenuto per venti anni a Roma in un'ottica di pedagogia sociale ${ }^{2}$. Il nucleo fondante di questo
\end{abstract}

1 Centro Shi Shu Bhavan che significa Casa del bambino in bengali: centro educativo per infanzia immigrata e romana.

2 Il progetto è nato con la cooperativa ASAL associazioni studi America Latina all'inizio degli anni '90 ed è proseguito, a partire dal 2000, con la gestione dell'associazione Zero in Condotta, ONLUS da me fondata. Il progetto è stato chiuso il 31 dicembre 2016, a causa dell'interruzione dei finanziamenti da parte del Comune di Roma, dopo infinite, inutili proteste di genitori, operatori e pochi altri centri simili attivi nel territorio romano. All'interno del progetto un bambino costava al comune meno di quanto non costasse un bambino in una scuola dell'infanzia o nido comunale o statale, ed i servizi erano molto più diversificati e integrati. Ma il progetto era gestito dal settore servizi sociali, che non è riuscito a trovare un accordo con l'ufficio dei servizi educativi e con il sindaco, per salvare questa esperienza.

* Università Degli Studi Roma Tre. Dipartimento Scienze della Formazione. Roma, Italia. E-mail: anna.aluffipentini@uniroma3.it. http://orcid.org/0000-0002-2037-5534 
progetto è il concetto di spazio potenziale di Winnicott $(1971,1986)$, applicato all'educazione interculturale e al sostegno alla genitorialità. Dati gli sviluppi delle politiche educative italiane attuali, ${ }^{3}$ alcune pratiche che sono state concretamente realizzate in questo progetto, grazie al costante accompagnamento di consulenza pedagogica, potranno essere recuperate per promuovere una dimensione di accoglienza delle diversità nel contesto formativo e per rendere l'istituzione scolastica, anche quella della prima infanzia, un contesto democratico in cui si promuove la parità tra $\mathrm{i}$ bambini.

Parole chiave: Educazione interculturale. Spazio potenziale. Accoglienza. Migrazione. Incubatore di continuità. Infanzia immigrata.

\section{RESUMO}

Nesta contribuição pretende-se dar conta da situação italiana quanto à abordagem educacional para a primeira infância e descrever um projeto intercultural ${ }^{4}$, concebido, implementado, monitorado e apoiado em Roma, por vinte anos, na visão da pedagogia social ${ }^{5}$. O núcleo fundador deste projeto é o conceito de espaço potencial de Winnicott (1971, 1986), aplicado à educação intercultural e ao apoio à parentalidade. Dada a evolução das atuais políticas educacionais italianas ${ }^{6}$, algumas práticas concretizadas neste projeto, graças ao acompanhamento constante de aconselhamento pedagógico, podem ser recuperadas para promover uma dimensão de acolhimento da diversidade no contexto educacional e fazer da instituição escolar, inclusive aquelas para a primeira infância, um contexto democrático no qual a igualdade entre crianças é promovida.

Palavras-chave: Educação intercultural. Espaço potencial. Acolhimento. Migração. Incubadora de continuidade. Infância imigrante.

3 Legge 13 aprile 2017, n. 65, sull'educazione dell'infanzia 0-6 anni.

4 Centro Shi Shu Bhavan que em bengali significa Casa para crianças: centro educacional para a infância romana e imigrada.

5 O projeto nasceu com cooperativa ASAL, Associação de Estudos da América Latina, no início dos anos 90 e continuou, a partir de 2000, com a gestão da "Associazione Zero in Condotta Onlus " fundada por mim. O projeto terminou em 31 de dezembro de 2016, devido à interrupção do financiamento pelo município de Roma, após inúmeros protestos sem sucesso dos pais, funcionários e alguns outros centros similares, em atividade no território romano. Dentro do projeto, uma criança custava para o município menos do que uma criança em um jardim de infância ou escola municipal ou estadual, e os serviços eram muito mais diversificados e integrados. Mas o projeto foi gerenciado pelo setor de serviços sociais, que não conseguiu chegar a um acordo com o escritório de serviços educacionais e o prefeito, para preservar essa experiência.

6 Lei n. 65, de 13 de abril de 2017, sobre a educação da infância de 0 a 6 anos. 


\begin{abstract}
This article seeks to highlight the Italian situation concerning a particular pedagogical early childhood approach by describing an intercultural project ${ }^{7}$ that was planned, implemented, monitored and supported from a social pedagogical perspective over the course of twenty years in Rome ${ }^{8}$. The principle concepts on which the project was based are that of the "potential space' (Winnicott 1971, 1989) applied to intercultural pedagogy and that of support for parenting skills. In view of current educational policies in Italy ${ }^{9}$ some forms of practice which were developed in the course of this project, thanks to the constant pedagogical support and consultancy it received, can be extrapolated in order to promote a pedagogical dimension that appreciates and welcomes diversity and helps educational institutions, including those of early childhood education, to become democratic environments that promote equality among children.
\end{abstract}

Keywords: Intercultural pedagogy. Early childhood education. Potential space. Migration. Incubator of continuity. Social integration.

\title{
Cura e apprendimento
}

La cura del bambino nella prima infanzia si basa in Italia, come in Europa e nei paesi industrializzati, su una conoscenza del bambino scientificamente fondata, sulla dimensione di diritto e su una definizione delle competenze educative.

L'apprendimento nella prima infanzia è legato imprescindibilmente all'accudimento e, nell'accudimento, la dimensione fisica della cura è preponderante.

Una parte della psicologia del ' 900 con Maslow (1954) ha gerarchizzato i bisogni; d'altro canto gli studi sulla deprivazione affettiva (Spitz, 1965) hanno

7 'Centro Shi Shu Bhavan' which means 'House of the Child' in Bengali, an early education centre for children with immigrant and Romany background.

8 The project originated from the work of the social cooperative ASAL (Associations of Latin American Studies) at the beginning of the 1990s and from 2000 onwards was managed by the non-profit association 'Zero in Condotta' which I founded. The project closed on 31 December 2016 when the City Council of Rome stopped financing it despite endless but ineffective protests by parents, operators and some other similar centres active in the territory of Rome. A child attending the centre actually cost the City Council less that the attendance at a public preschool or nursery would cost and the services offered within the ambit of the centre were much more diversified and integrated. But the project came under the aegis of the social services which were unable to negotiate an agreement with the educational services and the City Mayor that would have allowed to continue operating the centre.

9 Law No. 65 of 13 April 2017 on Childhood Education for the years 0-6. 
mostrato come sia difficile attribuire priorità a certi bisogni, dato che la relazione risulta essere importante almeno quanto il nutrimento: senza relazione viene meno la voglia di nutrirsi e senza nutrimento e relazione le performances cognitive vengono indebolite.

Se la gerarchizzazione dei bisogni di Maslow significa che senza mangiare non abbiamo voglia di imparare a leggere, ci troviamo tutti d'accordo. Ma sappiamo anche che il bambino apprende anche mentre mangia, e apprende MENTRE, e a causa del fatto che, viene tenuto, lavato, vestito, cambiato.

In Italia è stata da poco approvata una legge che riconosce l'educazione della fascia 0-3 anni come parte costitutiva del sistema formativo integrato. Ciò rientra nel generale orientamento europeo. Bisogna comunque tener presente che se nell'UE-28 quasi il 94\% dei bambini fra i 4 anni e l'età di inizio della scuola obbligatoria frequenta istruzione pre-primaria, in Italia il numero dei bambini sale al 98\%. Per quanto riguarda la fascia 0-3, la questione fino ad oggi è stata diversa: se è vero che solo il $30 \%$ dei bambini europei frequenta strutture ECEC, in Italia la percentuale si aggira intorno al 25\%, mentre siamo al $50 \%$ in Olanda, Svezia e Francia ed al 74\% in Danimarca. Il diritto all'ECEC sin dalla nascita esiste in Estonia, Slovenia, Finlandia, Svezia e Norvegia.

La nuova legge italiana garantisce sulla carta alle bambine ed ai bambini, dalla nascita fino ai sei anni, di sviluppare potenzialità di relazione, autonomia, creatività, di promuovere un apprendimento, in un adeguato contesto affettivo, ludico e cognitivo. La nuova legge vigila sulle pari opportunità di educazione $\mathrm{e}$ di istruzione, di cura, di relazione e di gioco, affermando che bisogna superare disuguaglianze e barriere territoriali, economiche, etniche e culturali.

Il sistema integrato di educazione e istruzione promuove quindi la continuità del percorso educativo e scolastico; concorre a ridurre gli svantaggi culturali, sociali e relazionali, e favorisce l'inclusione di tutte le bambine e di tutti i bambini. Gli ambiti nei quali la legge vuole promuovere uguaglianza tra bambini sono: la disabilità, la diversità culturale, il sostegno alla genitorialità, la conciliazione di tempi di lavoro e di cura, la formazione continua, il lavoro pedagogico territoriale.

Questo genere di approccio all'infanzia è un'opportunità per i bambini e le loro famiglie, ma può svilupparsi in due direzioni: portare ad una scolarizzazione eccessiva del nido o ad una descolarizzazione della scuola. Può portare a una istituzionalizzazione del bambino o ad un allestimento personalizzato e rassicurante del nido; ad una valorizzazione del genitore o ad un indebolimento del suo ruolo, ad un riconoscimento del bambino come persona anche spirituale o ad una reificazione dei suoi bisogni.

Il nuovo orientamento può portare alla formazione di professionisti rigidi, timorosi e politically correct, oppure ad avere operatori capaci di coinvolgere 
i bambini e le famiglie nel piacere di conoscersi, conoscere, imparare e divertirsi soppesando opportunità e rischi di un'educazione liberante, stimolante e rispettosa di ogni differenza.

Sappiamo bene che la situazione reale nelle istituzioni educative attuali in Italia non è tale da garantire standard qualitativi elevati a tutti e piena partecipazione dei bambini all'offerta educativa. Dietro al crescente numero di BES (soggetti con Bisogni Educativi Speciali) si cela in parte l'incapacità della scuola di promuovere davvero le potenzialità dell'infanzia.

È per questa ragione che intendiamo presentare un'esperienza del privato sociale che può costituire un modello operativo di riferimento per rendere accoglienti scuole e servizi sociali in relazione all'accoglienza dei migranti e di tutti i bambini. La mia convinzione corroborata dal lavoro sul campo è che la capacità di accogliere i diversi costituisca una insostituibile prova della qualità dei servizi educativi, anche per i cosiddetti uguali o normodotati (Aluffi Pentini, 1998).

\section{Shi Shu Bhavan: una scuola per tutti}

Il centro Shi Shu Bhavan si è proposto nella periferia romana come centro educativo interculturale per bambini dai due ai sei anni, con l'intento di accogliere, e poi inviare nelle scuole pubbliche - possibilmente dopo un solo anno, ma se necessario anche dopo quattro anni - i bambini che lo hanno frequentato. Il centro è stato frequentato per venti anni, ogni anno, da un minimo di venticinque bambini, provenienti da una trentina di paesi diversi.

Il centro può essere considerato un "incubatore di continuità"10 (Aluffi Pentini, 2002, 2008), vale a dire un luogo che accoglie, rassicura e rafforza persone spaesate, nel senso di persone private del loro paese di origine. Un incubatore, che a partire dall'accoglienza e dalla cura delle famiglie e dei loro figli, si adopera a ricreare una sorta di "fiducia di base" nel contesto che le circonda, sostenendo e incoraggiando così la possibilità di inserire il progetto migratorio in una cornice di significato personale e familiare, ma soprattutto esistenziale.

Il progetto pedagogico ha fatto riferimento al legame che Winnicott (1971, 1986) propone tra cultura e spazio potenziale e ha recuperato il significato di questo legame in relazione alla necessità dei migranti di acquisire una nuova

10 L'incubatore è quella macchina che consente al bambino prematuro di essere specialmente protetto fino al momento in cui diventa in grado di essere come tutti gli altri neonati nati a termine. 
fiducia di base nel nuovo contesto di vita. Il pedopsichiatra inglese sottolinea, infatti, l'importanza dello spazio potenziale tra il bambino e la madre come quello spazio che inizialmente mantiene una continuità con il legame prenatale $\mathrm{e}$, in generale, come uno spazio caratterizzato dalla fiducia di base. Successivamente, questo spazio, tra il bambino e la madre, si amplia per far posto all'esperienza del gioco e del gioco visto come esperienza culturale. Si tratta appunto dello spazio potenziale, spazio è definito da Winnicott sacro, perché è lo spazio sicuro al quale il bambino torna in seguito a nuove esperienze, e dove può mettere ciò che trova. Dove riesce ad integrare nuove esperienze in un contesto di fiducia già sperimentata. Grazie alle mie osservazioni sul campo di contesti educativi interculturali e grazie ai molti colloqui condotti negli anni con i genitori immigrati, ho toccato con mano quanto la "rinascita" del migrante nel paese di arrivo somigli a questa dinamica della prima infanzia di cui Winnicott parla. Lo spazio potenziale necessita, infatti, di essere ricostruito, per il migrante, dopo l'esperienza di discontinuità. Tale discontinuità è sperimentata nello spostamento dal paese di origine al paese verso il quale si emigra e si traduce spesso in spaesamento in termini psicologici, oltre che nel senso di concreto spaesamento (privazione del proprio paese). La rinascita è necessaria, ed è necessario sperimentare un nuovo spazio potenziale nella "nascita" nel paese di arrivo che risulta un mondo nuovo. Deve avvenire una rinascita psicologica che passa attraverso luoghi particolarmente accoglienti, dove si ricrea una fiducia di base. Per questa ragione c'è bisogno di "incubatori di continuità" per le famiglie immigrate: luoghi educativi accoglienti per i propri figli, luoghi che possano assolvere ad una necessaria funzione di rassicurazione, di riassicurazione. L'incubatore di continuità ricrea una fiducia di base allestendo un contesto sufficientemente buono ${ }^{11}$, predispone l'opportunità di vivere un nuovo processo di socializzazione che è paragonabile a quello delle prime esperienze di mondo che il bambino esperisce e che sono mediate dalla madre.

Il lavoro, che Shi Shu Bhavan ha svolto negli anni, è partito quindi dal doppio intento di essere per il bambino un luogo concreto in cui vivere, e, allo stesso tempo, per le famiglie, un luogo simbolico di riorganizzazione di un progetto di vita. Questo genere di dinamica diventa possibile se si crea un clima di fiducia e se il progetto educativo che riguarda il proprio figlio viene davvero condiviso tra famiglia immigrata e istituzione educativa.

L'incubatore di continuità, ovvero l'istituzione educativa così concepita, è quindi un luogo in cui si "arriva" nel paese ospite anche sul piano affettivo e simbolico, oltre che con il corpo e oltre che sul piano realistico e pragmaticamente inevitabile. Un luogo dove si sceglie di esserci.

11 Si fa qui riferimento al concetto di madre sufficientemente buona di Winnicott. 
Si tratta di un luogo che solleva dalla cura del figlio in modo da poter lavorare o cercare lavoro, ma, allo stesso tempo, di un luogo in cui si può essere sostenuti ed accompagnati nel mettere a punto un nuovo progetto di vita.

Tutto ciò richiede di predisporre uno spazio di accoglienza discreto e silenzioso in cui:

a) L'elemento linguistico non crei un sentimento di esclusione da parte di chi non padroneggia la lingua del paese di arrivo.

b) L'attenzione ai bisogni non significhi diventare pressanti per volerli individuare a tutti i costi.

c) La dimensione del tempo sia vista nell'ottica del concetto di spazio potenziale tra il bambino e la madre, vale a dire nella sua dimensione di pazienza atemporale, incondizionata.

d) L'ascolto silenzioso conservi una dimensione di ascolto attivo.

e) Si riconosca sia sul piano simbolico sia su quello pratico che ci vuole tempo per tradurre i bisogni in parole.

f) La rassicurazione sia trasmessa dallo sguardo prima ancora che dalle parole.

g) Gli operatori abbiano la capacità di rispecchiare la bellezza di chi hanno di fronte (bambino o genitore che sia) e le sue potenzialità.

h) Si trasmetta la fiducia nel fatto che lo stato di spaesamento è transitorio e che si sta intraprendendo un percorso di autonomia per gestire la propria vita nel nuovo contesto.

Infatti, bisogna tener conto che, spesso il genitore immigrato in un nuovo contesto, percepisce una propria inadeguatezza a far fronte a richieste non chiare e a concezioni educative diverse da quelle alle quali era abituato. Non ci si rende mai abbastanza conto che questa "inadeguatezza" non dipende dalle caratteristiche del genitore, bensì dalla nuova situazione contingente.

\section{La consulenza pedagogica interculturale}

Il servizio di consulenza pedagogica nel corso di anni di colloqui con i genitori ha raccolto numerose esperienze diversissime tra di loro, ma con un denominatore comune, vale a dire il bisogno di sentirsi accolti. Si è constatato come il costruirsi di un rapporto di fiducia con gli operatori risultasse rasserenante per la famiglia e positivo per il minore.

Pertanto si è definita una prassi di accoglienza reale e simbolica delle famiglie costituita da: a) colloquio iniziale con l'educatrice, per l'iscrizione; 
b) incontro di gruppo con i genitori per condividere il progetto educativo e le regole del contesto (anche, per esempio, per illustrare il lavoro di osservazione della psicopedagogista su tutti i bambini, che sarà poi oggetto di condivisione con i genitori); c) spiegazione del significato degli incontri individuali con la psicopedagogista (un incontro iniziale per tutti, e successivi incontri su richiesta degli operatori o del genitore); d) primo colloquio con la psicopedagogista; e) incontri tematici con i genitori; f) eventuali osservazioni su bambini con difficoltà particolari e eventuale invio ad altri professionisti.

Questo iter viene presentato come finalizzato alla costruzione di un progetto comune rivolto al benessere del bambino, alla sua ottimale integrazione nel gruppo dei pari e all'apprendimento graduale e non pressante della lingua italiana, mantenendo a casa l'utilizzo della lingua abitualmente parlata in famiglia.

Nel centro si è quindi instaurato nel corso degli anni un circolo virtuoso tra la pratica dei colloqui collettivi e individuali e la definizione nel corso delle riunioni di equipe dello stile educativo comune da adottare, per creare un ambiente rassicurante per i genitori e predisporre un luogo sereno in cui vivere per i bambini.

La consulenza pedagogica interculturale ha accompagnato famiglie, operatori ed equipe allargata ${ }^{12}$ e curato e orientato i rapporti con le istituzioni territoriali in un'ottica di community education. Si è tenuta sempre presente la necessità di creare dei ponti d'incontro con le istituzioni anche nel senso di insegnare alle famiglie a utilizzare i servizi ed a cogliere per sé e per i propri figli le opportunità che il territorio offre.

La consulenza è presentata alle famiglie sia nell'incontro di gruppo sia in quello individuale, come un'opportunità. Si condivide con regolarità il progetto educativo del centro e si illustra alle famiglie il significato del lavoro interculturale.

Si ascoltano poi i bisogni fondamentali delle famiglie, offrendo la possibilità di focalizzarsi sull'esperienza migratoria, ma non dando per scontato che il punto di partenza della consulenza sia la migrazione. Fondamentalmente s'incoraggiano le famiglie a fidarsi dei loro desideri e a credere nei loro progetti, invitandole a condividere la loro visione del mondo e a partecipare attivamente al progetto educativo.

Il progetto socio-educativo si arricchisce quindi progressivamente del sentire e della crescita di tutti. È in questo senso che il vivere creativo di cui Winnicott scrive, in merito alla nascita dell'esperienza culturale nel bambino, e che prende le mosse dall'abitare lo spazio di gioco tra il bambino e la madre,

12 Pediatra, presente periodicamente nel corso dell'anno per visitare tutti i bambini, assistenti sociali, servizi del territorio, psicoterapeuti dell'associazione convenzionata con il centro. 
concerne anche il vivere creativo della famiglia immigrata. La famiglia immigrata passa da essere oggetto di cura a soggetto capace di contribuire creativamente alla vita sociale. Il centro educativo frequentato dal figlio è un piccolo incubatore di tale creatività, uno spazio protetto nel quale sperimentarsi.

\section{Estendere a tutti l'esperienza interculturale}

Un simile approccio, sviluppato per favorire l'accoglienza interculturale, potrebbe risultare utilissimo anche per accompagnare le famiglie autoctone che hanno, per esempio, al nido, un primo contatto con le istituzioni educative e si aprono nella nuova esperienza genitoriale ad un nuovo mondo. Si inizia così a considerare ogni incontro educativo tra famiglie e operatori come ad un incontro interculturale, nel senso di promuovere una condivisione di idee educative finalizzata alla costruzione della fiducia e a lavorare ad un progetto comune sui bambini.

Alla luce di questa esperienza ventennale, l'orientamento che la nuova legge sull'infanzia in Italia propone, ossia l'intento di integrare cura, educazione e istruzione, può realizzarsi solo assumendo un'ottica di accoglienza progettuale e di rispecchiamento positivo, analoga a quella che, nel centro interculturale Shi Shu Bhavan, abbiamo sperimentato con successo per diversi anni.

Prima di chiudere il centro, abbiamo realizzato una semplice ricerca valutativa $^{13}$, contattando le famiglie che avevano frequentato il centro. Tutti coloro che sono stati rintracciati hanno affermato di avere, anche a distanza di anni, un buon ricordo del centro e di averlo consigliato ad altri, ma soprattutto che tutti i loro figli, indipendentemente dai risultati legati strettamente al profitto, avevano un buon rapporto con la scuola e in generale con il contesto italiano.

Ciò ha confermato la nostra convinzione di quanto sia importante creare uno spazio di accoglienza e di partecipazione anche per le famiglie, e quanto sia funzionale alla crescita del bambino creare un imprinting di relazione positiva tra genitori e operatori, primi rappresentanti di un'istituzione educativa italiana.

13 Sono state realizzate da un'operatrice esterna circa duecento telefonate. Hanno risposto una cinquantina di famiglie - i numeri di telefonia mobile cambiano spesso nei primi anni di immigrazione a seconda delle offerte più vantaggiose, anche per parlare con il proprio paese di origine - e un incontro con dieci mamme che avevano mandato i figli al centro nel corso degli anni. 


\section{Vivere creativo: un progetto satellite}

Il progetto socio-educativo del centro interculturale Shi Shu Bhavan è stato gestito in parallelo ad un progetto di accoglienza abitativa per nuclei familiari immigrati (madre - figli minori) ${ }^{14}$.

Nei locali di una delle case di accoglienza è stato realizzato un laboratorio artistico (pittura) aperto alle ospiti, alle ex ospiti delle case di accoglienza, e a mamme del quartiere, per dare loro l'opportunità di condividere momenti di creatività con i loro figli e con le operatrici.

Il progetto è durato circa sei mesi ${ }^{15}$, a cadenza settimanale, e ha coinvolto due gruppi di mamme a seconda dell'età dei figli (prima infanzia e adolescenza).

Il progetto è stato condotto da un pittore professionista ${ }^{16} \mathrm{e}$ ha prodotto opere collettive a tema libero, e focalizzate sul tema del viaggio.

Le mamme, richiedenti asilo, si sono messe in gioco e hanno condiviso le loro esperienze, anche con le operatrici come non era mai accaduto in situazioni più strutturate (interviste o questionari), pensate espressamente per conoscere il loro vissuto. Le mamme si sono sentite accolte incondizionatamente, non giudicate, e soggetti attivi nel territorio, dal momento che, nella "loro" casa, hanno ospitato nuclei familiari esterni al progetto. Da "ospiti-ospitate" sono diventate "ospiti-ospitanti".

Il loro apprezzamento per l'attività svolta è stato notevole. I lavori di pittura sono stati esposti nel corso di un evento organizzato dall'artista che ha condotto i laboratori e, per le mamme, il fatto di diventare visibili attraverso la loro produzione artistica è stato motivo di grande soddisfazione.

È parso inoltre che la coesione all'interno dei gruppi appartamento e il clima di fiducia, anche nei confronti degli operatori, migliorassero.

Data la brevità dell'esperienza, una valutazione del percorso realizzato non fornisce elementi certi per affermare che essa possa essere replicata con analoghi risultati, in contesti simili.

Ciò che però risulta chiaro è l'affinità di questo tipo di attività con il tema winnicottiano dell'esperienza culturale e artistica, e il legame di questa esperienza di spazio potenziale con la riflessione qui presentata in merito al progetto educativo del centro Shi Shu Bhavan.

14 Ammr Bhavan, che significa casa della mamma, accoglieva 25 persone tra mamme e figli. Il progetto è stato chiuso a settembre 2017, perché il tipo di finanziamento previsto non consentiva più di svolgere il lavoro di qualità realizzato precedentemente.

15 L'esiguità del finanziamento non ha consentito di prolungare l'esperienza, nonostante la generosità dell'artista che lo ha condotto, Carlo Gori, e i costi conseguentemente contenuti.

16 V. nota precedente. 
Aver creato un contesto protetto per le persone (madre - bambino) e per la relazione tra le persone (madre - bambini-operatrici), e aver proposto un'attività concreta di mediazione "culturale" e artistica, finalizzata alla promozione del vivere creativo, in un contesto interculturale, ha dato i suoi frutti e ha integrato l'esperienza di sostegno alla genitorialità portata avanti per anni nel centro interculturale per la prima infanzia.

\section{Contestualizzare le esperienze}

La legge sull'infanzia varata recentemente in Italia propone un'integrazione di accudimento, cura e istruzione a nostro avviso funzionale all'utilizzo degli spazi educativi come luoghi di accoglienza per le persone immigrate.

Per fare sì che tali spazi diventino anche luoghi di integrazione di esperienze culturali diversificate, è necessario ripensare la logica della trasmissione delle competenze, con la consapevolezza dell'esistenza di un patrimonio culturale spirituale e valoriale variegato, che può andare a costituire un "nuovo" patrimonio comune, se persone diverse vivono insieme in una dimensione creativa. Non si tratta più di insegnare in modo ripetitivo e acritico una cultura nazionale immutabile, ma di trovare attività da fare insieme che possano mediare lo scambio e la conoscenza reciproca.

Ciò richiede una particolare sensibilità sociale per cogliere bisogni diversi e bisogni che cambiano nel tempo, e richiede una riflessione sull'esperienza culturale nel significato primordiale che le viene attribuito da Winnicott.

Anche per quanto riguarda la formazione del personale che lavora con i bambini, l'approccio winnicottiano, che colloca l'esperienza culturale nello spazio potenziale della relazione materna, può fornire utili spunti di riflessione. In quest'ottica, ogni teoria educativa e ogni conoscenza che si acquisisce in merito alle pratiche educative va assimilata e personalizzata filtrandola nel sentire di chi lavora con il bambino.

Questo non significa lasciare spazio ad un'arbitrarietà scriteriata, ma comprendere che si può scongiurare il rischio di tecnicismi sterili di chi lavora con i bambini solo se si mantiene una genuinità e una consapevolezza di fondo rispetto alle proprie azioni, solo se si valorizza una colorazione emotiva forte nelle interazioni verbali e non, e se ci si confronta collegialmente a partire dalle situazione concrete in equipe o in supervisione. Nessuna teoria applicata in modo pedissequo, unilaterale e auto-referenziale, garantisce di fare il bene di tutti i bambini. Limitandosi alla teoria, l'educatore non vede i singoli bambini, 
non si auto-valuta a partire dalla interazioni che vive. Se l'educatore cerca solo di ripensare alle "istruzioni per l'uso del bambino dei manuali" non può percepirsi in una relazione viva. Un bambino non si diverte se l'educatore non si diverte, non ama parlare se l'educatore non ha il gusto di comunicare, non ama una lingua straniera (non impara la L2) se non condivide una dimensione vitale in quella lingua. Attraverso il filtro condiviso di un setting, curato, esplicito e dinamico, l'educatore è capace di riproporre uno spazio educativo transizionale e di accudire con il cuore, vagliando con cura i suoi gesti accudenti e comunicativi, sia nei confronti del bambino, sia nei confronti della famiglia, autoctona o alloctona che sia.

Se l'educatore entra in quest'ottica, sarà capace di declinare di volta in volta esigenze e situazioni contingenti, in funzione del rispetto dei bisogni sostanziali e fondamentali di cura.

Contestualizzare le pratiche significa accettare e assumere le dicotomie tra locale e globale, mediando tra i livelli possibili di composizione delle due istanze e riconoscere che anche criteri di qualità, norme di sicurezza e igienico sanitarie non sono universali ed assolute: le famiglie immigrate hanno bisogno di fiducia e gradualità per capire ed accettare le abitudini e le regole di un nuovo contesto. Le abitudini non sono scontate e non sono necessariamente il metro di valutazione per una genitorialità più o meno valida.

Dal famoso esempio del figlio di Gardner (1991) che gioca con la chiave in un albergo cinese, e che viene lasciato libero dai suoi genitori americani di esplorare, mentre per i cinesi che assistono alla scena sarebbe doveroso fargli vedere come si fa a infilare la chiave nel "modo giusto", capiamo quanto sia variabile il modo giusto di accompagnare la crescita del bambino: si può apprendere per prova ed errore o per imitazione; non esiste una cultura migliore in assoluto.

\section{Un po' di relativismo ma non troppo}

L'agenda 20-30 dell'ONU ci aiuta a riflettere sull'interdipendenza globale. Bisogna quindi riflettere su altre discrepanze culturali o paradossi. Bisogna riflettere sul paradosso di una casa sicura, un'aula sicura, dei giochi sicuri laddove si vive invece in un ambiente inquinato o nutrendosi di un cibo "avvelenato".

Bisogna riflettere su cosa vuol dire lo stile di vita di un bambino occidentale se lo confrontiamo a quello di un bambino delle bidonville. Perché la varietà delle situazioni deve farci contestualizzare il vero e il giusto. 
Ad esempio, quello che in un nido romano può essere un rapporto numerico inaccettabile tra adulti e bambini (o uno stile autoritario altrettanto inaccettabile) è assolutamente appropriato in un contesto dove l'istituzione educativa sovraffollata e autoritaria costituisce la salvezza per un bambino che altrimenti vivrebbe per strada: per un bimbo che condivide l'unico pasto quotidiano con altri cento bambini, facendo a turno per il piatto con i suoi compagni.

In una società, come quella italiana, dove ci sono più adulti che bambini e dove la natalità è bassa, l'educazione del bambino fuori dalla famiglia può essere vista come una sorta di istituzionalizzazione. Per un bambino africano che vive per strada, il nido risulta personalizzante e stimolante.

Rapporti numerici e standard formativi degli operatori sono dei fattori che incidono sull'educazione del bambino e ne influenzano le competenze, ma che devono essere contestualizzati. Come devono essere contestualizzate le competenze genitoriali.

Nella società globalizzata è bene abituarsi a relativizzare ogni forma di posizione drastica sull'educazione della prima infanzia.

Basta riflettere anche semplicemente sui cambi di mode del ventesimo secolo, sull'allattamento al seno o quello artificiale, sul cambiamento e/o abolizione dell'uso delle punizioni corporali nell'arco di un paio di generazioni in Europa. Gli studi interculturali sull'accudimento ad altro o basso contatto che ci dicono che, se nel mondo occidentale borghese la cameretta dove il bambino dorme da solo è un must, per molte madri "sufficientemente buone" di altre culture, dormire lontano dal proprio figlio è assolutamente impensabile e incompatibile con l'essere madre ${ }^{17}$.

\section{Conclusioni}

Le condizioni dell'infanzia sono molto variabili da un angolo all'altro del pianeta, ma attraverso la conoscenza e talvolta la deplorazione di tali condizioni, attraverso l'esperienza con vari bambini e esseri umani, noi ci formiamo un'idea di benessere e di contesto sufficientemente sano. In ogni luogo la competenza

17 Possiamo guardare anche all'interno dell'Europa alle normative che concernono l'organizzazione delle istituzioni della prima infanzia: In Gran Bretagna un educatore che lavora con bambini di due anni non può occuparsi di più di 4 bambini, in Lituania i bambini sono 15, a Cipro sono 16. In Finlandia sopra i 4 anni ci deve essere un educatore ogni 7, mentre a Cipro basta un educatore per 25. L'ampiezza dei gruppi di bambini di 4 anni in Germania e Slovacchia è di dieci, in Irlanda 26. In altri paesi non è definito al livello nazionale ma varia a livello comunale. 
educativa consiste nell'individuare azioni educative possibili, che salvaguardino al meglio un futuro vivibile e possibilmente godibile per ogni bambino. Tenendo d'occhio elaborate teorie, standard qualitativi elevati, ma anche la concretezza del qui ed ora. E tenendo d'occhio il bello, che a differenza del vero e del giusto, ha qualcosa di universale.

L'esperienza romana che ho descritto ha promosso per venti anni questo tipo di cura educativa interculturale e di competenze di accoglienza degli operatori. Gli operatori hanno lavorato con la prima infanzia e con famiglie diversissime tra di loro e hanno fornito un servizio di qualità che è fondamentale per TUTTI i bambini. Gli operatori hanno anche imparato a riflettere criticamente, a partire dalla quotidianità del luogo in cui i bambini vivono, e a confrontarsi sulle dinamiche di discriminazione che i bambini e le loro famiglie sperimentano nella società. Hanno imparato a coniugare un clima sereno e attività creative $\mathrm{e}$ divertenti, con il lavoro di empowerment delle famiglie e dei bambini stessi, in modo da rafforzarli, prima del passaggio ad altre istituzioni educative.

Coltivare questo tipo di educazione serve a riempire di contenuti le buone leggi e a diffondere la speranza di riuscire ad attuarle sempre di più. Quando un'esperienza educativa innovativa si conclude, in questo caso per incuria da parte delle istituzioni, ci si augura che qualcosa resti. Qualcosa può restare grazie alla diffusione delle idee di un progetto attraverso la scrittura e grazie a operatori che continuano a lavorare in altri contesti. Ma soprattutto qualcosa resta attraverso i bambini che hanno fatto un'esperienza positiva e che sono cresciuti. L'esperienza dei bambini che hanno frequentato Shi Shu Bhavan ha arricchito il patrimonio sociale e interculturale della città di Roma. I primi dati emersi da una ricerca ancora in corso sugli esiti del progetto lasciano pensare che le famiglie immigrate che hanno frequentato il centro abbiano tratto, da questa esperienza, la capacità di rapportarsi in modo sano alle istituzioni educative, che i loro figli si siano inseriti positivamente nelle scuole e che gli operatori, nei nuovi luoghi di lavoro, spendano le loro competenze, promuovendo una dimensione davvero interculturale del lavoro socioeducativo.

Il fine ultimo di ogni progetto che nasce per un target caratterizzato da fragilità sociale è che non ci sia più bisogno del progetto stesso, perchè la scuola e la società sono in grado di accogliere tutti allo stesso modo. Ci sembra tuttavia che ci sia ancora bisogno, almeno in Italia, di laboratóri di accoglienza, ovvero di spazi educativi che fanno della cura interculturale il fulcro della loro progettazione e che si strutturino come luoghi di vivere creativo e di empowerment sociale. 


\section{TESTI CITATI}

Aluffi Pentini, Anna (1998) L'intercultura fa bene alla scuola, Quaderni ISMU, Milano. Aluffi Pentini, Anna (2008) Der interkulturelle Kindergarten als Ort praktizierter Kinderrechte. Das Recht auf Wohlsein, in Eichelberger Harald., Elisabeth Furch (Hsg) Kulturen, Sprachen, Welten, Studien Verlag, Wien. pp. 7-18.

Gardner, Howard (1991) To Open Minds, Basic Books, New York.

Maslow, Abraham H. (1954) Motivation and Personality, Oxford.

Spitz, René (1965) The First Year of Life: A Psychoanalytic Study of Normal and Deviant Development of Object Relations, International Universities Press. (1972)

Winnicott, Donald W. (1986) Home Is Where We Start From. New York: W.W. Norton. Winnicott, Donald W. (1971) Playing and Reality. Middlesex, England: Penguin Books.

Testo ricevuto il 9 febbraio 2018 . Testo approvato il 12 febbraio 2018. 
